\title{
Use of gas liquid chromatography in combination with pancreatic lipolysis and multivariate data analysis techniques for identification of lard contamination in some vegetable oils
}

\begin{abstract}
A study was conducted to investigate the use of gas liquid chromatography (GLC) to identify lard (LD) contamination in palm oil (PO), palm kernel oil (PKO), and canola oil (CLO). Vegetable oils were deliberately adulterated with animal fats such as LD, beef tallow (BT), and chicken fat $(\mathrm{CF})$ in varying proportions. In order to monitor the fatty acid (FA) compositional changes due to adulteration, GLC analyses of fatty acid methyl esters (FAME) were performed on 2-monoacylglycerol (2-MG) and neutral triacylglycerol (TAG) isolated from each sample. For the evaluation of FA data, multivariate statistical techniques were employed. The results showed that canonical discriminant (CANDISC) analysis was the most effective technique for discriminating LD-adulterated samples from those adulterated with other animal fats. Additionally, mathematical equations obtained by simple regression analysis could be used for quantification of LD contents in admixtures.
\end{abstract}

Keyword: Adulteration, Animal fat, Gas liquid chromatography, Lard, Vegetable oils, Multivariate data analysis 